\title{
Compliance of a Small Convenience Sample and Efficacy of Short Term Modified Carbohydrate Diet on Weight Loss in Overweight College Students: A Pilot Study
}

\author{
Jay Kandiah, Dawn Brinson, Valerie Amend
}

Department of Family and Consumer Sciences, Ball State University, Muncie, USA.

Email: vaamend@bsu.edu

Received February 29 ${ }^{\text {th }}, 2012$; revised April $9^{\text {th }}, 2012$; accepted April $16^{\text {th }}, 2012$

\begin{abstract}
The purpose of this pilot study was to determine overweight students' compliance on low and moderate carbohydrate diets and its influence on weight loss. The 28 day study was divided into two experimental periods of 14 days each. For the first 14 days, Group $1(n=6)$ received LC diet (30 grams carbohydrate/day with ad libitum intake of protein and fat), and group $2(\mathrm{n}=8)$ received MC diet (60 grams carbohydrate/day with ad libitum intake of protein and fat). After 14 days, there was a crossover of the diets. Two random 24-hour diet records, urinary ketones, and daily emotional and physical well-being journals evaluated participants' dietary compliance. Height, weight, body mass index, and urine ketones were assessed at baseline, days 14 and 28. A $2 \times 2$ ANOVA was conducted to examine the difference between groups and to determine if a difference existed from baseline to the end of the diet period. During the study period, irrespective of carbohydrate levels, a vast majority of participants had above or below the recommended intake of carbohydrates, indicating non-compliance due to various reasons. During each experimental period, although weight loss differences between groups over time did not exist, there was a significant weight loss within subjects over time (p < 0.01). Presence of urinary ketones during the dietary interventions were not statistically significant. In conclusion, modified carbohydrate diets were effective with weight loss; however participants were non-compliant with their de- fined dietary protocols.
\end{abstract}

Keywords: Carbohydrate; Diet; Compliance; College Students; Weight Loss

\section{Introduction}

During the past 20 years there has been a dramatic increase in obesity in the United States (US). In 20072008 , the prevalence of obesity was $32.2 \%$ among adult men and 35.5\% among adult women [1]. In 2009, none of the states met the Healthy People 2010 obesity target of $15 \%$, and the self-reported overall prevalence of obesity among US adults had increased 1.1 percentage points from 2007 [1]. Health related problems of obesity include hypertension, hyperlipidemia, coronary heart disease, stroke, diabetes, cancer and psychological disorders [2].

Strategies that have been sought out for weight loss have been diet pills, diuretics, food supplements, skipping meals, fasting, laxative use, vomiting, exercising, and lower calorie intake [3]. While these have gained in popularity, obesity trends continue to rise. In part, failure to lose significant weight occurs because achieving weight loss through dieting or exercise requires maintenance of behavior change which is difficult to sustain [4].

Modified carbohydrate (CHO) diets are one example of current trends in weight loss. These diets contain less than the United States Department of Agriculture's (USDA) recommendation of $45 \%$ - $65 \%$ of total calories from carbohydrate (generally between $<10 \%$ to $30 \%$ of daily energy intake) [5]. The efficacy of low-CHO diets on weight loss has been attributed to several factors, including a satiating effect of fat and protein if individuals replace carbohydrates in their diet, increased energy expenditure in metabolism of protein and fat, appetite suppression, and reduction of caloric intake due to limiting of food choices [6-13]. Recent diets such as the Atkins diet minimize total carbohydrate consumption, but do not adjust fat intake or demand a calorie restriction [14]. Another popular trend is the South Beach diet, which has several phases and differing carbohydrate levels [15]. The main theory behind these diets is the restriction of carbohydrates will result in ketosis, increased lipolysis and decreased lipogenesis [14].

Dietary compliance with weight loss diets, specifically low or moderate $\mathrm{CHO}$, is crucial in achieving partici- 
pants' weight loss goals. To the best of the researchers' knowledge, little or no studies have looked specifically at compliance of modified $\mathrm{CHO}$ diets in the college population. Therefore, the purpose of this pilot study was to determine the effect of compliance level (using dietary recalls and ketone production as indicators) on low and moderate $\mathrm{CHO}$ diets and its effect on weight loss.

\section{Methods and Materials}

Prior to the start of the study, the research was reviewed and approved by the University's Institutional Committee on Investigation Involving Human Subjects.

For the purpose of this study, the low (LC) and moderate carbohydrate (MC) diets were 30 grams and 60 grams, respectively, with ad libitum intakes of protein and fat. This CHO regimen and timeline was selected since the recognized Atkins Diet's initial two week induction phase for weight loss recommends 20 grams CHO per day [14].

College students from a Midwestern University were recruited through the use of electronic mass email. Inclusion criteria were 18 - 26 years; body mass index (BMI) $\geq 25 \mathrm{~kg} / \mathrm{m}^{2}$; stable weight for at least one month; physicians' evaluation on health status; abstinence of dietary restrictions for three months prior to the research; absence of medications related to weight loss; free of health disorders/diseases; and avoidance of tobacco products. Upon recruitment into the study, additional criteria included exclusion of alcoholic beverages and nutrition supplements; limited caffeine intake (one, 6 - 8 fl.oz serving/day); and maintenance of usual physical activity.

Twenty-two subjects who met the above criteria volunteered to participate in the research. Prior to the start of the study, approximately one week, subjects were educated by a registered dietitian about food selection and reading nutrition labels. In addition, subjects were provided with a list of foods to limit/avoid during the study. Since the participants were college students, the researchers specified foods that were available in the dining services that they could consume to comply with the protocol. The dietitian was available via telephone, scheduled counseling hours and email throughout the study and met with the subjects on alternate days to answer questions and provide further education and reinforcement of dietary protocol. To ensure participants understanding of the dietary regimen, the registered dietitian quizzed the participants on foods and portion sizes. The 28-day study was divided into two experimental periods of 14 days each. The 14-day study period was chosen to allow participants sufficient time to appreciate any change in weight and to determine their capacity for following the diet. For the first 14 days, one-half of the subjects (Group 1) were instructed to follow the LC diet (30 grams carbohydrate/day with ad libitum intake of protein and fat)) and the other half (Group 2) were to adhere to the MC diet (60 grams carbohydrate/day with ad libitum intake of protein and fat). Each participant was responsible for controlling and recording their own CHO intake. After 14 days, there was a crossover of the diets (Table 1).

To aid in verifying adherence of the study protocol, subjects were asked to keep daily emotional and physical well-being journals, complete two random 24-hour diet records, which were analyzed using ESHA Food Processor Software 8.0 2005, and urinary ketones were assessed. Since this research study used ad libitum amounts of protein and fat, analysis of these macronutrients were not assessed. To assist subjects in accuracy of portion sizes, verbal, written, and photographic representations of portion sizes of common foods were provided. Daily behavioral and emotional logs were also collected (e.g. tobacco use, alcohol, supplements, medications, physical

Table 1. Experimental diet plan.

\begin{tabular}{|c|c|c|c|c|}
\hline Period & Days & Diet Type & \# of Subject & Day of Data Collection and Variables \\
\hline Baseline Data & & Ad Libitum & 14 & $\begin{array}{ll}\text { Day } 0 \\
\text { - } & \text { Height } \\
\text { - } & \text { Weight } \\
\text { - } & \text { Urine }\end{array}$ \\
\hline Experimental I Diet (LC-MC) & 14 Days & $\begin{array}{l}{ }^{*} \mathrm{LC} \\
{ }^{* *} \mathrm{MC}\end{array}$ & $\begin{array}{l}6(\mathrm{f}=6, \mathrm{~m}=0) \\
8(\mathrm{f}=6, \mathrm{~m}=2)\end{array}$ & $\begin{array}{ll}\text { Day } 14 \\
\text { - Weight } \\
\text { - } \quad \text { Urine }\end{array}$ \\
\hline Experimental II Diet (MC-LC) & 14 Days & $\begin{array}{l}\text { MC } \\
\text { LC }\end{array}$ & $\begin{array}{l}6(f=6, m=0) \\
8(f=6, m=2)\end{array}$ & $\begin{array}{l}\text { Day } 28 \\
\text { - Weight } \\
\text { - } \quad \text { Urine }\end{array}$ \\
\hline
\end{tabular}

Diet during the two experimental periods of 14 days each composed of: ${ }^{*}$ LC (Low-CHO Diet): 30 g CHO/day with ad libitum protein and fat intakes; ${ }^{* *}$ MC (Moderate-CHO Diet): $60 \mathrm{~g}$ CHO/day with ad libitum protein and fat intakes. 
activity).

Height, weight, BMI, and urine ketones were assessed at baseline, days 14, and 28. Height was recorded to the nearest. 25 inches using a stadiometer and weight was determined using SECA ${ }^{\mathrm{TM}}$ Digital Scale. Assessment of ketones was used to evaluate participants' dietary compliance as the production of ketone bodies is a normal response to a shortage of glucose (CHO) [16]. Urine samples were collected in a sterile container and assessed using Bayer Corporation Ketostix ${ }^{\circledR}$ [7]. Presence of ketones was defined as either negative $(0 \mathrm{mg} / \mathrm{dL})$, trace ( $5 \mathrm{mg} / \mathrm{dL})$, small $(15 \mathrm{mg} / \mathrm{dL})$, moderate $(40 \mathrm{mg} / \mathrm{dL})$, or large $(80-160 \mathrm{mg} / \mathrm{dL})$ and were correlated with the reference color chart.

Difference in body weight and urinary ketone production were compared in the two groups from baseline to the end of the first experimental period (14 days) and from the beginning of the second experimental period to the end of the second experimental period (14 days). A 2 $\times 2$ ANOVA was conducted to examine the difference between the groups and to determine if a difference existed from baseline to the end of the diet period. When violations of sphericity occurred, the Huynh-Feldt epsilon was used in the univariate analysis to compensate. A p-value of $<0.05$ was considered statistically significant.

\section{Results}

Fourteen of the original twenty-two subjects completed the 28-day study. Six of the participants were excluded due to various reasons, including inability to participate in the research due to personal reasons, newly diagnosed health issues, and newly prescribed medications that interfered with the study. All subjects were Caucasian Americans and their age ranged from 20 - 22 years. Two were males and 12 were females and everyone was classified as overweight (mean BMI > 25), none of the participants were obese.

During experimental period I, participants on the LC diet $(n=6)$ consumed an average of $46.4 \pm 32.4$ grams of CHO per day, above the recommended amount. Only one participant complied with the $\mathrm{CHO}$ allotment, while the others were above or below the recommended intakes. When Group 1 participants were crossed over to the MC diet, mean daily intake of carbohydrate was $62.7 \pm 35.5$ grams per day. None of the participants $(n=6)$ were in compliance with the $\mathrm{CHO}$ recommendations.

When Group 2 was placed on the LC diet, participants $(\mathrm{n}=8)$ consumed a mean CHO level of $55.1 \pm 30.6$ grams of CHO per day. Like their counterparts, with the exception of one participant $(13 \%)$, the others $(n=7)$ were above or below the recommended intake of $\mathrm{CHO}$. Similar observations were noted when participants were on MC diet. Although the mean intake of carbohydrate was $61.0 \pm 15.4 \mathrm{~g}$ CHO per day, all eight participants were above or below the recommended intakes.

Table 2 shows weight loss of participants between the two groups while following the LC and MC diets. In the current study, for each experimental diet, there was a significant weight loss within subjects over time ( $\mathrm{p}<$ 0.01 ). There was not, however, a significant difference in weight loss between the diet groups over time $\left(\mathrm{F}_{(1.61,19.37)}\right.$ $=39.391, \mathrm{p}=0.353$ ).

Urinary ketones were absent in both groups at baseline. After the first experimental diet period, 50\% of participants on LC (Group 1) and MC diets (Group 2) produced ketones. Following the second experimental period, urinary ketone levels of participants on MC (Group 1) and LC diets (Group 2) were 16\% vs $12.5 \%$ respectively, indicating no significant difference in urinary ketones were present. Table 3 shows the presence of urine ketones, measured as acetoacetic acid, over time, and the difference between groups.

\section{Discussion}

Results of the study revealed that during each experimental diet, within subjects over time, there was a sig-

Table 2. Weight loss in pounds between diet groups over time.

\begin{tabular}{|c|c|c|c|c|c|}
\hline Source & Type III Sum of Squares & $\mathbf{d f}^{1}$ & Mean Square & $\mathbf{F}$ & $\mathbf{p}$ \\
\hline \multicolumn{6}{|l|}{ Within Subjects } \\
\hline Time & 474.855 & 1.614 & 294.262 & 39.391 & $<0.001$ \\
\hline Time*Group & 12.726 & 1.614 & 7.886 & 1.056 & 0.353 \\
\hline Error (Time) & 144.659 & 19.365 & 7.470 & & \\
\hline \multicolumn{6}{|l|}{ Between Subjects } \\
\hline Group & 9.013 & 1 & 9.013 & .002 & 0.961 \\
\hline Error & 44360.576 & 12 & 3696.715 & & \\
\hline
\end{tabular}

${ }^{1}$ Huynh-Feldt correction. 
Table 3. Urine ketones over time and between groups.

\begin{tabular}{|c|c|c|c|c|c|}
\hline Source & Type III Sum of Squares & df & Mean Square & $\mathbf{F}$ & $\mathbf{p}$ \\
\hline \multicolumn{6}{|l|}{ Within Subjects } \\
\hline Time & 91.146 & 1 & 91.146 & 0.117 & 0.738 \\
\hline Time*Group & 441.146 & 1 & 441.146 & 0.566 & 0.466 \\
\hline Error (Time) & 9352.604 & 12 & 779.384 & & \\
\hline \multicolumn{6}{|l|}{ Between Subjects } \\
\hline Group & 193.527 & 1 & 193.527 & 0.105 & 0.751 \\
\hline Error & 22085.938 & 12 & 1840.495 & & \\
\hline
\end{tabular}

${ }^{1}$ Huynh-Feldt correction.

nificant weight loss. Weight loss may be attributed to depletion of glycogen stores, restriction of calories due to satiety effects of protein and fat, and/or the anorectic effect of ketosis. The findings of this research are congruent with previous researchers $[6,8-13,17,18]$. There was no significant difference in weight loss between diet groups (Groups 1 and 2) or between diet groups over time (14 and 28 days). This suggests that modifying and varying levels of CHO enable weight loss, regardless of level of restriction. Similarly, ketone production was not influenced by the dietary protocols.

According to Bray et al., CHO must be restricted to 50 g CHO/day to induce ketosis [6]. The current study showed no significant differences over time for ketone production, between diet groups, or between diet groups over time.

As stated previously, Group 1 participants while on LC diet consumed a mean intake of $46.39 \mathrm{~g} \mathrm{CHO/day,}$ which was greater than the recommended amount. Similarly, when on LC diet, Group 2 participants had mean daily intake of $55.08 \mathrm{~g} /$ day. These results indicate that the LC diet was impractical for participants to consume and comply. Although some subjects experienced ketosis, the majority did not, due to over consumption of CHO. A postulation could be that even though subjects selected and consumed foods from the list provided by the dietitian, there could have been poor compliance with regards to actual portion sizes eaten. Additionally, statistically significant differences were not likely to be observed from a small sample size. Foster et al., suggested that reduction in ketone production in their long term study (one year) with 63 obese men and women was due to poor patient compliance [7]. Moderate weight loss studies (6 months) reported by other researchers indicate greater compliance level was associated with greater weight loss. Corral et al. [19] examined the effect of diet and/or exercise and found dietary adherence is strongly associated with rates of weight loss and adversely affected by the severity of caloric restriction.

Adherence to long-term (one year) LC/high protein diets for weight loss with or without professional guidance indicated dietary adherence to treatment protocols were significantly associated with greater weight loss $[20,21]$. Non-compliance of varying CHO dietary regimens by the participants in the present study may be attributed to several factors; 1 ) demands and stress associated with college life influences food consumption choices and seeking high CHO comfort foods (sweet, chewy, doughy) [22,23]; 2) unpleasant symptoms (lethargy, irritability, hunger and stomach ache) reported by participants from their daily emotional and physical well-being journals led to diversion from prescribed diets (Table 4); 3) restrictive nature of the diet (30 or 60 grams $\mathrm{CHO}$ ) when compared to recommended amounts [5]; 4) lack of rapid weight loss; and 5) possibly due to the different lifestyle of the college student. It may have been easier for participants who lived in the dorm or in a confined metabolic unit to comply with the study compared to participants who lived in a house or apartment due to food cost and/or preparation.

This study was unique in several ways. Little to no studies associated with the college overweight population and their compliance to modified CHO diets have been investigated. Additional strengths of this research were that dietary protocols were more liberal than the traditional Atkins diet during induction phase thereby ena-

Table 4. Most common daily physical and well being traits reported by participants.

\begin{tabular}{ccc}
\hline & LC Diet & MC Diet \\
\hline 1. & Good (64\%) & Good (43\%) \\
2. & Tired (19\%) & Tired (31\%) \\
3. & Stomach Aches (13\%) & Hungry and Irritable (25\%) \\
\hline
\end{tabular}


bling participants in this study acted as their own controls. Finally, this research utilized the emotional and physical well-being journal to validate participants' adherence and reasons for non-compliance. As with most research studies, there were limitations present. The small sample size makes it difficult to generalize results to a larger population. Also, the short duration of the study period is a limiting factor.

With the increased prevalence of obesity, weight management health professionals are seeking effective interventions for clients' healthy and sustainable weight loss. Factors other than modified carbohydrate intakes should be taken into consideration. In spite of alterations in dietary carbohydrate protocol, significant weight loss may not occur if participants exhibit poor dietary compliance. Aggressive individual counseling in addition to support groups regarding dietary compliance (e.g., accurate assessment of food consumption, provision of nutrition education, and consistent adherence of dietary regimen at all times, etc.) could result in successful weight loss.

Future research should focus on using weighted food intake which would be more accurate and reflective of actual macronutrient consumption than estimated food intake. Using a larger sample size with different BMI levels would allow for greater generalization to US normal population. Development of long term study using varying levels of $\mathrm{CHO}$ and having a wash-out period between experimental diets would prevent any skewing of data and will assist in determining the efficacy of low CHO diets. A theoretical model, such as stage of change or health belief would possibly enrich the research as well.

\section{REFERENCES}

[1] Overweight and Obesity Trends among Adults, Center for Disease Control Web Site, 2010.

http://www.cdc.gov/nchs/pressroom/02news/obesityonris e.htm

[2] Overweight Prevalence, Center for Disease Control Web site, 2004.

http://www.cdc.gov/nchs/fastats/overwt.htm

[3] J. Kruger, D. A. Galuska, M. K. Serdula and D. A. Jones, "Attempting to Lose Weight: Specific Practices among US Adults," American Journal of Preventive Medicine, Vol. 26, No. 5, 2004, pp. 402-406. doi:10.1016/j.amepre.2004.02.001

[4] National Heart Blood and Lung Insitute, USDHHS Web-Site, 2011.

http://www.nhlbi.nih.gov/health/public/heart/obesity/lose _wt/behavior.htm

[5] Dietary Guidelines for Americans 2010, United States Department of Agriculture Web Site, 2011.

http://www.health.gov/dietaryguidelines/dga2010/dietary guidelines2010.pdf

[6] G. A. Bray, "Low-Carbohydrate Diets and Realities of Weight Loss,” JAMA, Vol. 289, No. 14, 2003, pp. 18531855. doi:10.1001/jama.289.14.1853

[7] G. D. Foster, H. R. Wyatt, J. O. Hill, B. G. McGuckin, C. Brill, S. Mohammed, P. O. Szapary, D. J. Rader, J. S. Edman and S. Klein, "A Randomized Trial of a LowCarbohydrate Diet for Obesity,” New England Journal of Medicine, Vol. 348, No. 21, 2003, pp. 2082-2090. doi:10.1056/NEJMoa022207

[8] J. C. Larosa, A. Gordon, R. Muesing and D. R. Rosing, "Effects of High-Protein, Low-Carbohydrate Dieting on Plasma Lipoproteins and Body Weight,” JADA, Vol. 77, No. 3, 1980, pp. 264-270.

[9] F. F. Samaha, N. Iqbal, P. Seshadri, K. L. Chicano, D. A. Daily, J. McGrory, T. Williams, M. Williams, E. J. Gracely and L. Stern, "A Low-Carbohydrate as Compared with a Low-Fat Diet in Severe Obesity,” New England Journal of Medicine, Vol. 348, 2003, pp. 2074-2081. doi:10.1056/NEJMoa022637

[10] L. Stern, N. Iqbal, P. Seshadri, K. L. Chicano, D. A. Daily, J. McGrory, M. Williams, E. J. Gracely, F. F. Samaha, "The Effects of Low-Carbohydrate versus Conventional Weight Loss Diets in Severely Obese Adults: One Year Follow Up of a Randomized Trial," Annals of Internal Medicine, Vol. 140, No. 10, 2004, pp. 778-786.

[11] D. M. Bravata, L. Sanders, J. Huang, H. M. Krumholz, I. Olkin, C. Gardner and D. M. Bravata, "Efficacy and Safety of Low-Carbohydrate Diets: A Systematic Review," JAMA, Vol. 289, 2003, pp. 1837-1850. doi:10.1001/jama.289.14.1837

[12] B. J. Brehm, R. J. Seeley, S. R. Daniels, D. A. D’Alessio, "A Randomized Trial Comparing a Very Low Carbohydrate Diet and a Calorie Restricted Low Fat Diet on Body Weight and Cardiovascular Risk Factors in Healthy Women," Journal of Clinical Endocrinology \& Metabolism, Vol. 88, No. 4, 2003, pp. 1617-1623. doi:10.1210/jc.2002-021480

[13] M. A. Denke, "Metabolic Effects of High-Protein, LowCarbohydrate Diets," American Journal of Cardiology, Vol. 88, No. 1, 2001, pp. 59-61. doi:10.1016/S0002-9149(01)01586-7

[14] J. Stanley, "Long-Term Adverse Health Effects of Atkins Diet Are Not Due to High Fat Content but Rather to High Protein Content,” Lipid Technology, 2003.

[15] A. Agatston, "The South Beach Diet: The Delicious, Doctor-Designed, Foolproof Plan for Fast and Healthy Weight Loss,” Rodale, Inc., New York.

[16] Living with Diabetes. American Diabetes Association Web-Site, 2012.

http://www.diabetes.org/living-with-diabetes/complicatio ns/ketoacidosis-dka.html

[17] S. T. St. Jeor, B. V. Howard, E. Prewitt, V. Bovee, T. Bazzarre and R. Eckel, "Dietary Protein and Weight Reduction: A Statement for Healthcare Professionals from the Nutrition Committee of the Council on Nutrition, Physical Activity, and Metabolism of the American Heart Association,” Circulation, Vol. 106, No. 20, 2001, pp. 
1869-1874. doi:10.1161/hc4001.096152

[18] G. Wright, B. Dawson, G. Jalleh and S. Law, "Impact of Compliance on Weight Loss and Health Profile in a Very Low Energy Program,” Australian Family Physician, Vol. 39, No. 1-2, 2010, pp. 49-52.

[19] P. Corral, P. Chandler-Laney, K. Casazza, B. Gower and G. Hunter, "Effect of Dietary Adherence with or without Exercise on Weight Loss: A Mechanistic Approach to a Global Problem,” Journal of Clinical Endocrinology \& Metabolism, Vol. 94, No. 5, 2009, pp. 1602-1607. doi:10.1210/jc.2008-1057

[20] S. Acharya, O. Elci, S. Sereika, E. Music, M. Styn, M. Turk and L. Burke, “Adherence to a Behavioral Weight Loss Treatment Program Enhances Weight Loss and Improvements in Biomarkers," Patient Preference and Adherence, Vol. 3, 2009, pp. 151-160.
[21] G. D. Brinkworth, M. Noakes, J. B. Koegh, N. D. Luscombe, G. A. Wittert, P. M. Clifton, "Long-Term Effects of a High Protein, Low Carbohydrate Diet on Weight Control and Cardiovascular Risk Markers in Obese Hyperinsulinemic Subjects," International Journal of Obesity, Vol. 28, 2004, pp. 661-670. doi:10.1038/sj.ijo.0802617

[22] J. Kandiah, M. Yake and H. Willet, "Effects of Stress on Eating Practices among Adults," Family and Consumer Sciences Research Journal, Vol. 37, No. 1, 2008, pp. 27-38. doi:10.1177/1077727X08322148

[23] J. Kandiah, M. Yake, M. Meyer and J. Jones, "Stress Influences Appetite and Comfort Food Preferences in College Women," Nutrition Research, Vol. 26, No. 3, 2006, pp. 118-123. doi:10.1016/j.nutres.2005.11.010 